\title{
Dissipativity analysis and stabilization for discontinuous delayed complex-valued networks via matrix measure method
}

\author{
Zengyun Wang ${ }^{1,2^{*}}$, Jinde $\mathrm{CaO}^{2^{*}}$ and Zhenyuan $\mathrm{Guo}^{3}$
}

\author{
"Correspondence: \\ sjyzywang@hnfnu.edu.cn; \\ jdcao@seu.edu.cn \\ 1 School of Mathematics and \\ Computational Science, Hunan First \\ Normal University, Changsha, China \\ ${ }^{2}$ The Jiangsu Provincial Key \\ Laboratory of Networked Collective \\ Intelligence, and School of \\ Mathematics, Southeast University, \\ Nanjing, China \\ Full list of author information is \\ available at the end of the article
}

\begin{abstract}
In this paper, we discuss the dissipativity and stabilization problems via matrix measure strategy. First, we propose a way to construct complex-valued set-valued maps and give the basic framework of complex-valued differential inclusions. In addition, based on matrix measure strategy and the generalized Halanay inequality, we analyze the dissipativity of the addressed discontinuous complex-valued neural networks by using two different ways. Furthermore, we design a set of controllers to guarantee the exponential stability of the studied networks. The main contribution of this paper is an extension of the dissipativity results of traditional neural networks to discontinuous ones. Finally, we give numerical examples to substantiate the merits of the obtained results.
\end{abstract}

Keywords: Complex-valued networks; Dissipativity analysis; Differential inclusion; Matrix measure method; Generalized Halanay inequality

\section{Introduction}

During the past ten years, numerous papers were published to present some results on dynamics of complex-valued systems due to their wide applications in imaging processing, speech synthesis, data fusion, and dynamical programming [1-5]. Many advantages of complex-valued networks, such as powerful new capabilities and computing, have been shown in [6]. The first advantage is that complex-valued neural networks can significantly improve the generalization capabilities. For example, the authors proposed a simple structure of complex-valued neural networks, which can efficiently deal with several nonlinear data separation problems at a high symbol rate. In fact, a complex-valued neural network can learn and estimate the rotation and scaling in the two-dimensional space. Therefore there exist several storage models for complex-valued neural networks at the same time [7]. Though there are many good properties of complex-valued neural networks, it is difficult to design the activation functions. As we know, we usually choose a differential and the bounded function as an activation function in a traditional neural network. However, every analytic and bounded function in the complex plane is constant [8]. Therefore, as a weaker choice, plenty of continuous nonanalytic functions and discontinuous functions become important candidates in designing complex-valued neural networks.

(c) The Author(s) 2018. This article is distributed under the terms of the Creative Commons Attribution 4.0 International License (http://creativecommons.org/licenses/by/4.0/), which permits unrestricted use, distribution, and reproduction in any medium, provided you give appropriate credit to the original author(s) and the source, provide a link to the Creative Commons license, and indicate if changes were made. 
In the past five years, many results on dynamics, including the existence and stability of equilibria and bifurcation analysis, of continuous complex-valued networks have been obtained [9-23]. However, all these mentioned results were obtained under the hypothesis of Lipschitz continuity. In fact, as shown by Hopfield [24, 25], the discontinuity is very common in mathematical models in many applied fields. In the following years, some dynamical behaviors, such as stability and periodic solutions, of discontinuous real-valued systems were extensively studied [26-34] via differential inclusion theory. In recent years, a small quantity of dynamical results, including multi-stability and $\mu$-stability, have been obtained for piecewise continuous complex-valued neural networks (CVNNs) [35-38]. However, all these results did not consider the problem that the equilibria happen to fall on the boundaries of the discontinuous functions. Based on our knowledge, there is almost no result on dynamics of complex-valued networks with general discontinuous right-side functions. Since differential inclusion theory is an efficient way to deal with discontinuous problems, to discuss discontinuous complex-valued differential problems, we need to propose a framework of complex-valued differential inclusion theory correspondingly. This is the first motivation of this paper.

It is known that an important issue of dynamics of a neural network is dissipativity because of its wide applications in stability theory and control theory [39-45]. Compared with the definition of Lyapunov stability, the notion of dissipativity is more general because the dissipativity analysis is aimed at an attractive set. As we know, we only need to analyze the dynamics of attractive set since it contains an equilibrium point, periodic solution, or chaos attractor [46-52]. Dissipativity theory also provides a fundamental framework to study control problems of neural networks. The notion of stabilization is that controllers can be added to neural networks to guarantee the stability of equilibria even though the original neural network has no equilibrium point or unstable one. During the past decades, numerous results on dissipativity analysis of real-valued systems, such as switching systems, fuzzy neural networks, digital filters, and discontinuous neural networks, have been obtained [33, 47-52]. For example, the dissipativity of switching systems and fuzzy neural networks are obtained by Shi [51], respectively. Moreover, the authors of [51] extend the dissipativity results to digital filters systems. Recently, the dissipativity analysis of complex-valued networks of fractional order was studied in [53]. However, according to what we know, the issue of dissipativity analysis and stabilization of discontinuous delayed complex-valued networks are not yet considered.

To study the dynamic behavior of CVNNs, many methods were proposed, such as the complex-valued Lyapunov function method and the synthesis method [11, 16, 17, 54, 55]. In [54] and [55], the author studied the stability of CVNNs via synthesis method. However, this method usually is used to analyze discrete models. Later, the direct Lyapunov function method is used to study the stability of CVNNs [11, 16, 17]. However, all these methods are difficult to deal with discontinuous CVNNs since no proper theoretical tools can be used to deal with complex-valued Lyapunov functions. In recent years, the matrix measure method is widely used to study the stability of neural networks in [33,56-58]. Since a matrix measure can be negative definite, which leads to less restrictive than matrix norm in handling matrix inequalities. Moreover, as we know, it is difficult to give a candidate function of discontinuous neural networks with multi-variable activation functions. The Lyapunov function is not necessary to construct by using a matrix measure strategy. For example, in [57], the authors studied the exponential stability of complex-valued net- 
works with continuous function. In [58], the exponential stability of nonlinear continuous complex-valued differential equations is studied via the matrix measure method. However, as far as we know, there are no dynamical results on discontinuous CVNNs via the matrix measure method. Moreover, separating a CVNN into real ones and direct analysis are two different strategies to deal with complex-valued problems. For example, these two methods are used to study the stability of complex-valued delayed neural networks in [9] and [11], respectively. Thus, what is the difference of the obtained results if we study the same dynamical behavior of CVNNs by these two strategies? This is the second motivation of this paper.

Based on the matrix measure theory, we discuss the dissipativity and stabilization of discontinuous delayed CVNNs by using the mentioned different strategies. Compared with the existing results, the most great improvement is removing the hypothesis of Lipschitz continuity of the activation function. We list the main contributions of this paper:

(1) We give an appropriate solution definition for a delayed complex-valued differential equation with discontinuous right-side by extending the real-valued functional differential inclusion theory.

(2) We propose a sufficient condition to ensure the global dissipativity of discontinuous complex-valued networks by using a complex-valued matrix measure.

(3) By separating the real and imaginary parts of discontinuous neural networks, we present a sufficient condition to realize global dissipativity by using the matrix measure method. This result is more general than the existing dissipativity results on real-valued neural networks.

(4) We design state-dependent controllers with switching term to guarantee the exponential stability of the equilibria of the studied network model.

\section{Neural network model and some preliminaries}

The discontinuous delayed complex-valued network model is given as follows:

$$
\dot{w}(t)=-D w(t)+A h(w(t))+B h(w(t-\tau))+J,
$$

where $w(t)=\left[w_{1}(t), w_{2}(t), \ldots, w_{n}(t)\right]^{\mathrm{T}} \in \mathbb{C}^{n}$ denotes the state vector, $D=\operatorname{diag}\left\{d_{1}, d_{2}, \ldots, d_{n}\right\}$ is a self-inhibition matrix with $d_{i}>0, A \in \mathbb{C}^{n \times n}$ and $B \in \mathbb{C}^{n \times n}$ show the connection weight matrices at times $t$ and $t-\tau$, respectively, $h(w(t))=\left[h_{1}\left(w_{1}(t)\right), h_{2}\left(w_{2}(t)\right), \ldots, h_{n}\left(w_{n}(t)\right)\right]^{\mathrm{T}} \in$ $\mathbb{C}^{n}$ denotes the complex-valued vector activation function, and $J=\left[J_{1}, J_{2}, \ldots, J_{n}\right]^{\mathrm{T}} \in \mathbb{C}^{n}$ is an input vector.

To further study the dynamics, we make the following two hypotheses on the function $h_{i}\left(w_{i}\right)$.

$A(1)$ For $k=1,2, \ldots, s, h_{i}\left(w_{i}\right)$ is continuous in finitely many open domains $E_{k}^{i}$ and discontinuous at $\partial E_{k}^{i}$; $E_{k}^{i}$ satisfies $\bigcup_{k=1}^{s}\left(E_{k}^{i} \cup \partial E_{k}^{i}\right)=\mathbb{C}$ and $E_{l}^{i} \cap E_{k}^{i}=\emptyset$ for $1 \leq l \neq k \leq s$. Moreover, the limit $h_{i k}\left(w_{0}\right)=\lim _{w \rightarrow w_{0}, w \in E_{k}^{i}} h_{i}(w)$ exists for all $w_{0} \in \partial E_{k}^{i}$.

$A(2)$ There are two constants $\alpha_{i} \geq 0$ and $\beta_{i} \geq 0$ such that

$$
\sup _{\gamma_{i} \in K\left[h_{i}\left(w_{i}\right)\right]}\left\|\gamma_{i}\right\| \leq \alpha_{i}\left\|w_{i}\right\|+\beta_{i}
$$

where $K\left[h_{i}\left(w_{i}\right)\right]=\left\{\sum_{k=1}^{s} \alpha_{k} h_{i k}\left(w_{i}\right) \mid \alpha_{k} \geq 0, \sum_{k=1}^{s} \alpha_{k}=1\right\}$ for $w_{i} \in \partial E^{i}$ and $K\left[h_{i}\left(w_{i}(t)\right)\right]=$ $h_{i}\left(w_{i}\right)$ for $w_{i} \in E^{i}$, where $\partial E^{i}=\bigcup_{k=1}^{s} \partial E_{k}^{i}$ and $E^{i}=\bigcup_{k=1}^{s} E_{k}^{i}$. 
Remark 1 Frankly speaking, $A(2)$ is an extension of the Lipschitz continuity condition, which is required in many papers $[9,15,58]$. In general, the constant $\beta_{i} \neq 0$ in $A(2)$ because of the discontinuity of the functions $h_{i}$. Here, we extend the neuron activation functions of system (2.1) to be discontinuous.

Since system (2.1) is a discontinuous network model, the classic theory of differential equations is out of work. Therefore one of the most important problems is giving an appropriate definition of a solution for a discontinuous system.

Consider the complex-valued differential equation

$$
\dot{w}(t)=h\left(t, w_{t}(\theta)\right)
$$

with the historical state $w_{t}(\theta)=w(t+\theta)$, where $\tau$ is a given positive number, $h: \mathbb{R} \times C \mapsto \mathbb{C}^{n}$ is essentially locally bounded and measurable, where $C$ is the space of continuous functions from $[-\tau, 0]$ to $\mathbb{C}^{n}$. Of course, $h\left(t, w_{t}(\theta)\right)$ may be discontinuous with respect to $w_{t}(\theta)$.

Since $h\left(t, w_{t}(\theta)\right)$ is a discontinuous function, we explain the definition of a solution for discontinuous differential equation (2.3). To give a definition of a solution for system (2.3), we introduce the following set-valued mapping definition.

Definition 2.1 For any $w \in E \subseteq \mathbb{C}^{n}$, if there exists a nonempty set $H(w) \in \mathbb{C}^{n}$ corresponding to $w$, then $w \mapsto H(w)$ is called a set-valued map. Furthermore, if for any $v_{0} \in E$ and any open set $N$ containing $H\left(w_{0}\right)$, there exists a neighborhood $M$ of $w_{0}$ such that $H(M) \subset N$, where $H(M)=\bigcup_{y \in M} H(y)$, then the set-valued map $H$ with nonempty values is upper semicontinuous at $w_{0}$.

Definition 2.2 For discontinuous system (2.3), we define

$$
H\left(t, w_{t}\right)=\bigcap_{\delta>0} \bigcap_{\mu(\mathbb{N})=0} K\left[h\left(t, B\left(w_{t}, \delta\right) \backslash \mathbb{N}\right)\right]
$$

where $K(\cdot)$ denotes the convex closure, $B\left(w_{t}, \delta\right)=\left\{w_{t}^{*} \in C \mid\left\|w_{t}^{*}-w_{t}\right\| \leq \delta\right\}$, and $\mu$ is the Lebesgue measure.

Definition 2.3 A complex-valued function $w(t): I \mapsto \mathbb{C}^{n}$ is a solution of system (2.3) if it is absolutely continuous on $\left[t_{1}, t_{2}\right] \subseteq I$ and satisfies $\dot{w}(t) \in H\left(t, w_{t}(\theta)\right)$ for almost all $t \in I$.

In the following, by taking advantage of the above definitions of differential inclusion, we introduce the following definitions of a solution to a discontinuous complex-valued neural network of system (2.1). Before doing this, we first introduce the definition of absolute continuity and measurability of set-valued map.

Definition 2.4 A function $w(t):[a, b] \mapsto \mathbb{C}$ is absolutely continuous if for any positive number $\varepsilon>0$, there exists $\delta>0$ such that $\sum_{i=1}^{n}\left\|w\left(b_{i}\right)-w\left(a_{i}\right)\right\|<\varepsilon$ for any $\left(a_{i}, b_{i}\right) \subseteq[a, b]$ satisfying $\sum_{i=1}^{n}\left(b_{i}-a_{i}\right)<\delta$.

Definition 2.5 A complex set-valued map $H:[a, b] \rightarrow \mathbb{C}^{n}$ is measurable if the nonnegative function $t \mapsto \operatorname{dist}(w, H(t))=\inf \{\|w-u\|, u \in H(t)\}$ is measurable for any $w \in \mathbb{C}^{n}$. 
Definition 2.6 A complex-valued function $w(t)$ defined on $[-\tau, T)$ is a solution of system (2.1) on $[-\tau, T)$ if

(i) $w(t)$ is continuous on $[-\tau, T)$ and absolutely continuous on any compact subinterval of $[0, T)$, and

(ii) for almost all $t \in[0, T)$, w(t) satisfies

$$
\dot{w}(t) \in-D w(t)+A K[h(w(t))]+B K[h(w(t-\tau))]+J \triangleq H\left(t, w_{t}\right) .
$$

It is easy to verify that the differential inclusion $H\left(t, w_{t}\right)$ is nonempty and compact convex. Moreover, $H\left(t, w_{t}\right)$ is upper semicontinuous and measurable in the sense of Definition 2.5. According to the measurable selection theorem in [59], there is a bounded measurable function $\gamma=\left[\gamma_{1}, \gamma_{2}, \ldots, \gamma_{n}\right]^{\mathrm{T}}$ satisfying

$$
\dot{w}=-D w+A \gamma+B \gamma_{\tau}+J
$$

for a.e. $t \geq 0$, where $\gamma_{i} \in K\left[h_{i}\left(w_{i}\right]\right.$ and $\gamma_{\tau}=\gamma(t-\tau)$. By Definition 2.6, $w$ is a solution of system (2.1), and $\gamma$ is an output solution corresponding to $w$.

In the following, we mainly consider the global dissipativity of discontinuous complexvalued networks (2.1) via the matrix measure method. Before doing so, let us give the definition of global dissipativity and matrix measure.

Definition 2.7 The network model (2.1) is globally dissipative if for any initial valued $\left(t_{0}, w_{0}\right)$, we can find a compact set $S \in \mathbb{C}^{n}$ and $T\left(w_{0}\right)$ such that the state solution $w\left(t, t_{0}, w_{0}\right) \in S$ for $t>t_{0}+T\left(w_{0}\right)$. Furthermore, if $w\left(t, t_{0}, w_{0}\right) \in S$ for all $w_{0} \in S$ and $t>T$, then the set $S$ is a forward invariant.

Definition $2.8([33,58])$ The matrix measure corresponding to a given matrix norm $\|A\|_{\mathcal{P}}$ is defined as

$$
\mu_{\mathcal{P}}(A)=\lim _{\Delta t \rightarrow 0^{+}} \frac{\|I+\Delta t A\|_{\mathcal{P}}-1}{\Delta t}
$$

where $I$ is the identity matrix.

We now introduce an important lemma named generalized Halanay inequalities, which will be used later.

Lemma 2.9 ([60]) For any nonnegative function $W(t)$ defined on $(-\infty,+\infty)$, if there exist three continuous functions $r(t) \geq 0, q(t) \geq 0, p(t) \leq 0$ and a positive number $\sigma$ such that

$$
D^{+} W(t) \leq r(t)+p(t) W(t)+q(t) \sup _{t-\tau \leq s \leq t} W(s), \quad t \geq t_{0}
$$

and

$$
q(t)+p(t) \leq-\sigma
$$


for $t \geq t_{0}$, then we have

$$
W(t) \leq \frac{r^{*}}{\sigma}+\left(\sup _{-\infty \leq s \leq t_{0}} W(s)-\frac{r^{*}}{\sigma}\right) e^{-\mu^{*}\left(t-t_{0}\right)},
$$

where $r^{*}=\sup _{t_{0} \leq s \leq+\infty} r(t), \quad \mu^{*}=\inf _{t \geq t_{0}}\left\{\mu(t)+p(t)+q(t) e^{\mu(t) \tau}\right\}$ and $D^{+} W(t)=$ $\varlimsup_{\lim _{\triangle t \rightarrow 0^{+}}} \frac{W(t+\Delta t)-W(t)}{\Delta t}$.

\section{Global dissipativity analysis}

Theorem 3.1 If the discontinuous functions of system (2.1) satisfy hypotheses $A(1)-A(2)$, then for any initial values, there exists at least one solution $w(t)$ defined on $[0,+\infty)$.

Proof According to our discussion, the complex-valued map $w(t) \mapsto H\left(t, w_{t}\right)$ is upper semicontinuous, and $H\left(t, w_{t}\right)$ is nonempty compact convex. By analysis similar to that in [61, Thm. 1, p. 77] the local solution $w(t)$ of (2.4) can be guaranteed.

From formula (2.2) we obtain that there exist two nonnegative constants $\bar{\alpha}$ and $\bar{\beta}$ such that

$$
\|K[h(w(t))]\|\|\leq \bar{\alpha}\| w(t) \|+\bar{\beta} .
$$

It follows that

$$
\begin{aligned}
\left\|H\left(t, w_{t}\right)\right\| & =\|-D w(t)+A K[h(w(t))]+B K[h(w(t-\tau))]+J\| \\
& \leq\|D\|\|w(t)\|+\|A\|(\bar{\alpha}\|w(t)\|+\bar{\beta})+\|B\|(\bar{\alpha}\|w(t-\tau)\|+\bar{\beta})+\|J\| \\
& =(\|D\|+\bar{\alpha}\|A\|)\|w(t)\|+\bar{\alpha}\|B\|\|w(t-\tau)\|+(\bar{\beta}(\|A\|+\|B\|)+\|J\|) \\
& =\overline{\bar{\alpha}}\|w(t)\|+\overline{\bar{\beta}}\|w(t-\tau)\|+\overline{\bar{\eta}},
\end{aligned}
$$

where $\overline{\bar{\alpha}}=\|D\|+\bar{\alpha}\|A\|, \overline{\bar{\beta}}=\bar{\alpha}\|B\|$, and $\overline{\bar{\eta}}=\bar{\beta}(\|A\|+\|B\|)+\|J\|$.

According to (2.4), for fixed $t$, we obtain

$$
w(t) \in w(0)+\int_{0}^{t} H\left(s, w_{s}\right) \mathrm{d} s .
$$

It follows that

$$
\begin{aligned}
\|w(t)\| & \leq \sup _{s \in[-\tau, 0]}\|w(s)\|+\int_{0}^{t}\left\|H\left(s, w_{s}\right)\right\| \mathrm{d} s \\
& \leq\left(\sup _{s \in[-\tau, 0]}\|w(s)\|+\overline{\bar{\eta}} t\right)+\overline{\bar{h}} \int_{0}^{t}(\|w(s)\|+\|w(s-\tau)\|) \mathrm{d} s,
\end{aligned}
$$

where $\overline{\bar{h}}=\max \{\overline{\bar{\alpha}}, \overline{\bar{\beta}}\}$. Since

$$
w(t-\tau) \in w(0)+\int_{0}^{t-\tau} H\left(s, w_{s}\right) \mathrm{d} s,
$$

we obtain

$$
\|w(t-\tau)\| \leq\left(\sup _{s \in[-\tau, 0]}\|w(s)\|+\overline{\bar{\eta}} t\right)+\overline{\bar{h}} \int_{0}^{t}(\|w(s)\|+\|w(s-\tau)\|) \mathrm{d} s .
$$


From our analysis and the Gronwall inequality we have

$$
\|w(t)\| \leq\|w(t)\|+\|w(t-\tau)\| \leq\left(2 \sup _{s \in[-\tau, 0]}\|w(s)\|+2 \overline{\bar{\eta}} t\right) e^{2 \overline{\bar{h}} t}
$$

According to the continuation theorem [61], $w(t)$ is defined on $[0,+\infty)$ and satisfies

$$
\dot{w}(t) \in-D w(t)+A K[h(w(s))]+B K[h(w(t-\tau))]+J .
$$

In the following, we consider the global dissipativity of discontinuous CVNNs (2.1) via the matrix measure method.

Theorem 3.2 If the discontinuous functions of system (2.1) satisfy hypotheses $A(1)-A(2)$ and there exists $\mu_{\mathcal{P}}(\cdot)$ satisfying

$$
\mu_{\mathcal{P}}(-D)+\|A\|_{\mathcal{P}}\|\alpha\|_{\mathcal{P}}+\|B\|_{\mathcal{P}}\|\alpha\|_{\mathcal{P}} \leq-\sigma<0
$$

where $\alpha=\operatorname{diag}\left(\alpha_{1}, \alpha_{2}, \ldots, \alpha_{n}\right)$, then system (2.1) is globally dissipative. Moreover, for any sufficiently small number $\varepsilon>0$,

$$
\mathcal{S}=\left\{v \in \mathbb{C}^{n}:\|z\|_{\mathcal{P}} \leq \frac{r}{\sigma}+\varepsilon\right\}
$$

is a globally positive attractive invariant set, where $r=\left(\|A\|_{\mathcal{P}}+\|B\|_{\mathcal{P}}\right)\|\beta\|_{\mathcal{P}}+\|J\|_{\mathcal{P}}$ and $\beta=\operatorname{diag}\left(\beta_{1}, \beta_{2}, \ldots, \beta_{n}\right)$.

Proof We choose the positive radially unbounded function $W(t)$ in Lemma 2.9 to be $\|w\|_{\mathcal{P}}$. Calculating $D^{+} W(t)$ along the trajectory of (2.5), we have

$$
\begin{aligned}
D^{+} W(t)= & \varlimsup_{\Delta t \rightarrow 0^{+}} \frac{\|w(t+\Delta t)\|_{\mathcal{P}}-\|w(t)\|_{\mathcal{P}}}{\Delta t} \\
= & \varlimsup_{\Delta t \rightarrow 0^{+}} \frac{\|w+\Delta t \dot{w}+o(\Delta t)\|_{\mathcal{P}}-\|w\|_{\mathcal{P}}}{\Delta t} \\
= & \varlimsup_{\Delta t \rightarrow 0^{+}} \frac{\left\|w+\Delta t\left[-D w+A \gamma+B \gamma_{\tau}+J\right]+o(\Delta t)\right\|_{\mathcal{P}}-\|w\|_{\mathcal{P}}}{\Delta t} \\
\leq & \varlimsup_{h \rightarrow 0^{+}} \frac{\|w+\Delta t(-D) w\|_{\mathcal{P}}-\|w\|_{\mathcal{P}}}{\Delta t}+\|A \gamma\|_{\mathcal{P}}+\left\|B \gamma_{\tau}\right\|_{\mathcal{P}}+\|J\|_{\mathcal{P}} \\
\leq & \varlimsup_{h \rightarrow 0^{+}} \frac{\|I+\Delta t(-D)\|_{\mathcal{P}}-1}{\Delta t}\|w\|_{\mathcal{P}}+\|A \gamma\|_{\mathcal{P}}+\left\|B \gamma_{\tau}\right\|_{\mathcal{P}}+\|J\|_{\mathcal{P}} \\
\leq & \mu_{\mathcal{P}}(-D)\|w\|_{\mathcal{P}}+\|A\|_{\mathcal{P}}\left(\|\alpha\|_{\mathcal{P}}\|w\|_{\mathcal{P}}+\|\beta\|_{\mathcal{P}}\right) \\
& +\|B\|_{\mathcal{P}}\left(\|\alpha\|_{\mathcal{P}}\left\|_{\tau}\right\|_{\mathcal{P}}+\|\beta\|_{\mathcal{P}}\right)+\|J\|_{\mathcal{P}} \\
\leq & \left(\mu_{\mathcal{P}}(-D)+\|A\|_{\mathcal{P}}\|\alpha\|_{\mathcal{P}}\right)\|w\|_{\mathcal{P}}+\|B\|_{\mathcal{P}}\left\|_{\alpha}\right\|_{\mathcal{P}}\left\|w_{\tau}\right\|_{\mathcal{P}} \\
& +\left(\|A\|_{\mathcal{P}}+\|B\|_{\mathcal{P}}\right)\|\beta\|_{\mathcal{P}}+\|J\|_{\mathcal{P}} .
\end{aligned}
$$


Let $p=\mu_{\mathcal{P}}(-D)+\|A\|_{\mathcal{P}}\|\alpha\|_{\mathcal{P}}, q=\|B\|_{\mathcal{P}}\|\alpha\|_{\mathcal{P}}$, and $r=\left(\|A\|_{\mathcal{P}}+\|B\|_{\mathcal{P}}\right)\|\beta\|_{\mathcal{P}}+\|J\|_{\mathcal{P}}$. Then by Lemma 2.9 and inequality (3.2) we obtain

$$
\|w\|_{\mathcal{P}}=W(t) \leq \frac{r}{\sigma}+\left(\sup _{-\infty \leq s \leq 0} W(s)-\frac{r}{\sigma}\right) e^{-\mu^{*} t}
$$

where $\mu^{*}$ is the solution of the equation $\mu+p+q e^{\mu \tau}=0$. Thus, for any sufficiently small $\varepsilon>0$, there exists $T>0$ such that

$$
\|w\|_{\mathcal{P}} \leq \frac{r}{\sigma}+\varepsilon, \quad \forall t>T
$$

Remark 2 In [58], the exponential stability on continuous complex-valued differential equations is studied by using the matrix measure method. However, there are almost no results on the dynamics of a discontinuous complex-valued network model via matrix measure method.

Suppose $w^{*}$ is an equilibrium of system (2.1) with continuous function, that is, $\beta_{i}=0$ in formula (2.2). We obtain the following corollary, which is an extensional result of [57] and [58].

Corollary 3.3 The continuous complex-valued system (2.1) is globally exponentially stable if there is $\mu_{\mathcal{P}}(\cdot)$ such that

$$
\mu_{\mathcal{P}}(-D)+\|A\|_{\mathcal{P}}\|\alpha\|_{\mathcal{P}}+\|B\|_{\mathcal{P}}\|\alpha\|_{\mathcal{P}} \leq-\sigma<0
$$

where $\alpha=\operatorname{diag}\left(\alpha_{1}, \alpha_{2}, \ldots, \alpha_{n}\right)$.

In the following, we study the global dissipativity of system (2.1) by transforming it into the corresponding real-valued system. Let $w=u+\mathbf{i} v, A=A^{R}+\mathbf{i} A^{I}, B=B^{R}+\mathbf{i} B^{I}, h(w)=$ $h^{R}(u, v)+\mathbf{i} h^{I}(u, v), \gamma(t)=\gamma^{R}+\mathbf{i} \gamma^{I}$, and $J=J^{R}+\mathbf{i} J^{I}$. Then system (2.1) and system (2.5) can be transformed to

$$
\left\{\begin{array}{l}
\dot{u}=-D u+A^{R} h^{R}(u, v)-A^{I} h^{I}(u, v)+B^{R} h^{R}\left(u_{\tau}, v_{\tau}\right)-B^{I} h^{I}\left(u_{\tau}, v_{\tau}\right)+J^{R}, \\
\dot{v}=-D v+A^{I} h^{R}(u, v)+A^{R} h^{I}(u, v)+B^{I} h^{R}\left(u_{\tau}, v_{\tau}\right)+A^{R} h^{I}\left(u_{\tau}, v_{\tau}\right)+J^{I},
\end{array}\right.
$$

and

$$
\left\{\begin{array}{l}
\dot{u}=-D u+A^{R} \gamma^{R}-A^{I} \gamma^{I}+B^{R} \gamma_{\tau}^{R}-B^{I} \gamma_{\tau}^{I}+J^{R} \\
\dot{v}=-D v+A^{I} \gamma^{R}+A^{R} \gamma^{I}+B^{I} \gamma_{\tau}^{R}+B^{R} \gamma_{\tau}^{I}+J^{I}
\end{array}\right.
$$

respectively, where the functions $h^{R}(u, v)$ and $h^{I}(u, v)$ are discontinuous in $R^{2 n}$.

From assumption $\mathrm{A}(1)$ we obtain that $h_{i}^{R}(u, v)$ and $h_{i}^{I}(u, v)$ are continuous at $E_{k}$ and discontinuous at $\partial E_{k} ; E_{k} \cap E_{l}=\emptyset$ for $k \neq l$, and $\bigcup_{k=1}^{s}\left(E_{k} \cup \partial E_{k}\right)=\mathbb{R}^{2}$. Furthermore, the limits $\lim _{(u, v) \rightarrow\left(u_{0}, v_{0}\right)} h_{i}^{R}(u, v)=h_{i}^{k R}\left(u_{0}, v_{0}\right)$ and $\lim _{(u, v) \rightarrow\left(u_{0}, v_{0}\right)} h_{i}^{I}(u, v)=h_{i}^{k I}\left(u_{0}, v_{0}\right)$ exist, where 
$(u, v) \in E_{k}$ and $\left(u_{0}, v_{0}\right) \in \partial E_{k}$. According to $\mathrm{A}(2)$, there exist $\alpha_{i}^{R}, \beta_{i}^{R}, \eta_{i}^{R}, \alpha_{i}^{I}, \beta_{i}^{I}$, and $\eta_{i}^{I}$ such that

$$
\left|\gamma_{i}^{R}\right| \leq \alpha_{i}^{R}\left|u_{i}\right|+\beta_{i}^{R}\left|v_{i}\right|+\eta_{i}^{R}, \quad\left|\gamma_{i}^{I}\right| \leq \alpha_{i}^{I}\left|u_{i}\right|+\beta_{i}^{I}\left|v_{i}\right|+\eta_{i}^{I}
$$

Remark 3 By our analysis, assumption $A(2)$ is more general than assumptions in many papers; see, for example, $[9,14]$. In fact, a discontinuous activation function becomes a possible choice in mathematical model of describing complex-valued network problems because of Liouville's theorem [8]. Differently from many papers, such as [11, 13, 15, 17, 19], since $h_{i}^{R}$ and $h_{i}^{I}$ are discontinuous activation functions, it is obvious that $\partial h^{R}(u, v) / \partial u$, $\partial h^{R}(u, v) / \partial v, \partial h^{I}(u, v) / \partial u$ and $\partial h^{I}(u, v) / \partial v$, may not exist.

Remark 4 It is obvious that $g_{i}^{R}$ and $g_{i}^{I}$ are bivariate functions and the definitions of differential inclusions in the previous literature are not valid; see [30-34, 60, 62]. Fortunately, the defined differential inclusions with bivariate functions under assumptions $A(1)-A(2)$ is an extension of the case of single-variable functions.

Remark 5 According our analysis, we know that the bivariate functions $h_{i}^{R}$ and $h_{i}^{I}$ are nonmonotone in variables $x$ and $y$, which are allowed to be unbounded. Therefore, the neuron activation functions $h_{i}^{R}$ and $h_{i}^{I}$ are more general than the functions in the existing results, such as $[28,30,33,34,60,62]$.

Theorem 3.4 If the discontinuous functions satisfy hypotheses $A(1)$ and $A(2)$ and there is a matrix measure $\mu_{\mathcal{P}}(\cdot)$ satisfying

$$
\mu_{\mathcal{P}}(-D)+\|\bar{A}\|_{\mathcal{P}} \max \left\{\|\alpha\|_{\mathcal{P}},\|\beta\|_{\mathcal{P}}\right\}+\|\bar{B}\|_{\mathcal{P}} \max \left\{\|\alpha\|_{\mathcal{P}},\|\beta\|_{\mathcal{P}}\right\} \leq-\sigma<0
$$

where $r=\left(\|\bar{A}\|_{\mathcal{P}}+\|\bar{B}\|_{\mathcal{P}}\right)\|\eta\|_{\mathcal{P}}+\|\bar{J}\|_{\mathcal{P}},\|\bar{A}\|_{\mathcal{P}}=\left\|A^{R}\right\|_{\mathcal{P}}+\left\|A^{I}\right\|_{\mathcal{P}},\|\bar{B}\|_{\mathcal{P}}=\left\|B^{R}\right\|_{\mathcal{P}}+\left\|B^{I}\right\|_{\mathcal{P}}$, and $\|\bar{J}\|_{\mathcal{P}}=\left\|J^{R}\right\|_{\mathcal{P}}+\left\|J^{I}\right\|_{\mathcal{P}}$, then the network model (2.1) is globally dissipative. Furthermore, for any sufficient small positive number $\varepsilon$,

$$
\mathcal{S}=\left\{w \in \mathbb{C}^{n}:\|\operatorname{Re}(w)\|_{\mathcal{P}}+\|\operatorname{Im}(w)\|_{\mathcal{P}} \leq \frac{r}{\sigma}+\varepsilon\right\}
$$

is a globally attractive positive invariant set.

Proof We consider the auxiliary function $W(t)=\|u\|_{\mathcal{P}}+\|u\|_{\mathcal{P}}$. Calculating $D^{+} W(t)$ along the trajectory of system (3.6), we have

$$
\begin{aligned}
D^{+} W(t)= & \varlimsup_{\Delta t \rightarrow 0^{+}} \frac{\|u(t+\Delta t)\|_{\mathcal{P}}-\|u(t)\|_{\mathcal{P}}}{\Delta t}+\varlimsup_{\Delta t \rightarrow 0^{+}} \frac{\|v(t+\Delta t)\|_{\mathcal{P}}-\|v(t)\|_{\mathcal{P}}}{\Delta t} \\
\leq & \varlimsup_{\Delta t \rightarrow 0^{+}} \frac{\|u+\Delta t(-D) u\|_{\mathcal{P}}-\|u\|_{\mathcal{P}}}{\Delta t}+\left\|A^{R} \gamma^{R}\right\|_{\mathcal{P}}+\left\|A^{I} \gamma^{I}\right\|_{\mathcal{P}} \\
& +\left\|B^{R} \gamma_{\tau}^{R}\right\|_{\mathcal{P}}+\left\|B^{I} \gamma_{\tau}^{I}\right\|_{\mathcal{P}}+\left\|J^{R}\right\|_{\mathcal{P}}+\varlimsup_{\Delta t \rightarrow 0^{+}} \frac{\|v+\Delta t(-D) v\|_{\mathcal{P}}-\|v\|_{\mathcal{P}}}{\Delta t} \\
& +\left\|A^{I} \gamma^{R}\right\|_{\mathcal{P}}+\left\|A^{R} \gamma^{I}\right\|_{\mathcal{P}}+\left\|B^{I} \gamma_{\tau}^{R}\right\|_{\mathcal{P}}+\left\|B^{R} \gamma_{\tau}^{I}\right\|_{\mathcal{P}}+\left\|J^{I}\right\|_{\mathcal{P}}
\end{aligned}
$$




$$
\begin{aligned}
& \leq \varlimsup_{\Delta t \rightarrow 0^{+}} \frac{\|I+\Delta t(-D)\|_{\mathcal{P}}-1}{\Delta t}\|u\|_{\mathcal{P}}+\left\|A^{R} \gamma^{R}\right\|_{\mathcal{P}}+\left\|A^{I} \gamma^{I}\right\|_{\mathcal{P}} \\
& +\left\|B^{R} \gamma_{\tau}^{R}\right\|_{\mathcal{P}}+\left\|B^{I} \gamma_{\tau}^{I}\right\|_{\mathcal{P}}+\left\|J^{R}\right\|_{\mathcal{P}} \\
& +\varlimsup_{\Delta t \rightarrow 0^{+}} \frac{\|I+\Delta t(-D)\|_{\mathcal{P}}-1}{\Delta t}\|v\|_{\mathcal{P}}+\left\|A^{I} \gamma^{R}\right\|_{\mathcal{P}}+\left\|A^{R} \gamma^{I}\right\|_{\mathcal{P}}+\left\|B^{I} \gamma_{\tau}^{R}\right\|_{\mathcal{P}} \\
& +\left\|B^{R} \gamma_{\tau}^{I}\right\|_{\mathcal{P}}+\left\|J^{I}\right\|_{\mathcal{P}} \\
& \leq \mu_{\mathcal{P}}(-D)\|u\|_{\mathcal{P}}+\left\|A^{R}\right\|_{\mathcal{P}}\left(\left\|\alpha^{R}\right\|_{\mathcal{P}}\|u\|_{\mathcal{P}}+\left\|\beta^{R}\right\|_{\mathcal{P}}\|v\|_{\mathcal{P}}+\left\|\eta^{R}\right\|_{\mathcal{P}}\right) \\
& +\left\|A^{I}\right\|_{\mathcal{P}}\left(\left\|\alpha^{I}\right\|_{\mathcal{P}}\|u\|_{\mathcal{P}}+\left\|\beta^{I}\right\|_{\mathcal{P}}\|v\|_{\mathcal{P}}+\left\|\eta^{I}\right\|_{\mathcal{P}}\right) \\
& +\left\|B^{R}\right\|_{\mathcal{P}}\left(\left\|\alpha^{R}\right\|_{\mathcal{P}}\left\|u_{\tau}\right\|_{\mathcal{P}}+\left\|\beta^{R}\right\|_{\mathcal{P}}\left\|v_{\tau}\right\|_{\mathcal{P}}+\left\|\eta^{R}\right\|_{\mathcal{P}}\right) \\
& +\left\|B^{I}\right\|_{\mathcal{P}}\left(\left\|\alpha^{I}\right\|_{\mathcal{P}}\left\|u_{\tau}\right\|_{\mathcal{P}}+\left\|\beta^{I}\right\|_{\mathcal{P}}\left\|v_{\tau}\right\|_{\mathcal{P}}+\left\|\eta^{I}\right\|_{\mathcal{P}}\right)+\left\|J^{R}\right\|_{\mathcal{P}}+\mu_{\mathcal{P}}(-D)\|v\|_{\mathcal{P}} \\
& +\left\|A^{I}\right\|_{\mathcal{P}}\left(\left\|\alpha^{R}\right\|_{\mathcal{P}}\|u\|_{\mathcal{P}}+\left\|\beta^{R}\right\|_{\mathcal{P}}\|v\|_{\mathcal{P}}+\left\|\eta^{R}\right\|_{\mathcal{P}}\right) \\
& +\left\|A^{R}\right\|_{\mathcal{P}}\left(\left\|\alpha^{I}\right\|_{\mathcal{P}}\|u\|_{\mathcal{P}}+\left\|\beta^{I}\right\|_{\mathcal{P}}\|v\|_{\mathcal{P}}+\left\|\eta^{I}\right\|_{\mathcal{P}}\right) \\
& +\left\|B^{I}\right\|_{\mathcal{P}}\left(\left\|\alpha^{R}\right\|_{\mathcal{P}}\left\|u_{\tau}\right\|_{\mathcal{P}}+\left\|\beta^{R}\right\|_{\mathcal{P}}\left\|v_{\tau}\right\|_{\mathcal{P}}+\left\|\eta^{R}\right\|_{\mathcal{P}}\right) \\
& +\left\|B^{R}\right\|_{\mathcal{P}}\left(\left\|\alpha^{I}\right\|_{\mathcal{P}}\left\|u_{\tau}\right\|_{\mathcal{P}}+\left\|\beta^{I}\right\|_{\mathcal{P}}\left\|v_{\tau}\right\|_{\mathcal{P}}+\left\|\eta^{R}\right\|_{\mathcal{P}}\right)+\left\|J^{I}\right\|_{\mathcal{P}} \\
& \leq \mu_{\mathcal{P}}(-D)\left(\|u\|_{\mathcal{P}}+\|v\|_{\mathcal{P}}\right)+\left(\left\|A^{R}\right\|_{\mathcal{P}}+\left\|A^{I}\right\|_{\mathcal{P}}\right)\left(\left\|\alpha^{R}\right\|_{\mathcal{P}}+\left\|\alpha^{I}\right\|_{\mathcal{P}}\right)\|x(t)\|_{\mathcal{P}} \\
& +\left(\left\|A^{R}\right\|_{\mathcal{P}}+\left\|A^{I}\right\|_{\mathcal{P}}\right)\left(\left\|\beta^{R}\right\|_{\mathcal{P}}+\left\|\beta^{I}\right\|_{\mathcal{P}}\right)\|v\|_{\mathcal{P}}+\left(\left\|B^{R}\right\|_{\mathcal{P}}\right. \\
& \left.+\left\|B^{I}\right\|_{\mathcal{P}}\right)\left(\left\|\alpha^{R}\right\|_{\mathcal{P}}+\left\|\alpha^{I}\right\|_{\mathcal{P}}\right)\left\|u_{\tau}\right\|_{\mathcal{P}} \\
& +\left(\left\|B^{R}\right\|_{\mathcal{P}}+\left\|B^{I}\right\|_{\mathcal{P}}\right)\left(\left\|\beta^{R}\right\|_{\mathcal{P}}+\left\|\beta^{I}\right\|_{\mathcal{P}}\right)\left\|v_{\tau}\right\|_{\mathcal{P}} \\
& +\left(\left\|A^{R}\right\|_{\mathcal{P}}+\left\|A^{I}\right\|_{\mathcal{P}}+\left\|B^{R}\right\|_{\mathcal{P}}+\left\|B^{I}\right\|_{\mathcal{P}}\right)\left(\left\|\eta^{R}\right\|_{\mathcal{P}}+\left\|\eta^{I}\right\|_{\mathcal{P}}\right)+\left\|J^{R}\right\|_{\mathcal{P}}+\left\|J^{I}\right\|_{\mathcal{P}} \\
& \leq\left\{\mu_{\mathcal{P}}(-D)+\|\bar{A}\|_{\mathcal{P}} \max \left\{\|\alpha\|_{\mathcal{P}},\|\beta\|_{\mathcal{P}}\right\}\right\}\left(\|u\|_{\mathcal{P}}+\|v\|_{\mathcal{P}}\right) \\
& +\|\bar{B}\|_{\mathcal{P}} \max \left\{\|\alpha\|_{\mathcal{P}},\|\beta\|_{\mathcal{P}}\right\}_{t-\tau \leq s \leq t} \max _{t}\left(\|u(s)\|_{\mathcal{P}}+\|v(s)\|_{\mathcal{P}}\right) \\
& +\left(\|\bar{A}\|_{\mathcal{P}}+\|\bar{B}\|_{\mathcal{P}}\right)\|\eta\|_{\mathcal{P}}+\|J\|_{\mathcal{P}} \text {. }
\end{aligned}
$$

Let $p=\mu_{\mathcal{P}}(-D)+\|\bar{A}\|_{\mathcal{P}} \max \left\{\|\alpha\|_{\mathcal{P}},\|\beta\|_{\mathcal{P}}\right\}, q=\|\bar{B}\|_{\mathcal{P}} \max \left\{\|\alpha\|_{\mathcal{P}},\|\beta\|_{\mathcal{P}}\right\}$, and $r=\left(\|\bar{A}\|_{\mathcal{P}}+\right.$ $\left.\|\bar{B}\|_{\mathcal{P}}\right)\|\eta\|_{\mathcal{P}}+\|\bar{J}\|_{\mathcal{P}}$. By Lemma 2.9 and inequality (3.8) we obtain

$$
\|u\|_{\mathcal{P}}+\|v\|_{\mathcal{P}}=W(t) \leq \frac{r}{\sigma}+\left(\sup _{-\infty \leq s \leq 0} W(s)-\frac{r}{\sigma}\right) e^{-\mu^{*} t},
$$

where $\mu^{*}$ is the solution of the equation $\mu+p+q e^{\mu \tau}=0$. Therefore, for any sufficient small $\varepsilon>0$, there exists $T>0$ such that

$$
\|\operatorname{Re}(w)\|_{\mathcal{P}}+\|\operatorname{Im}(w)\|_{\mathcal{P}} \leq \frac{r}{\sigma}+\varepsilon, \quad \forall t>T .
$$

Remark 6 In [57], the authors present some sufficient conditions to ensure the stability of a continuous complex-valued network, where $h(w)=h^{R}(\operatorname{Re}(w))+\mathbf{i} h^{I}(\operatorname{Im}(w))$. Here, we assume that the activation function has the form $h(w)=h^{R}(\operatorname{Re}(w), \operatorname{Im}(w))+\mathbf{i} h^{I}(\operatorname{Re}(w), \operatorname{Im}(w))$, which is more general than that in [57]. Furthermore, the function $h(w)$ may be nonLipschitz continuous or even discontinuous. 
Remark 7 Compared with the existing results, we extend global dissipativity results to real-valued discontinuous networks with bivariate function. If all imaginary parts of system (2.1) equal zero, then the results of Theorem 3.4 degenerate into Theorem 2 in [33], which presents sufficient conditions for the global dissipativity of a discontinuous network with univariate function.

Suppose $w^{*}=u^{*}+\mathbf{i} v^{*}$ is an equilibrium of (2.1) and $\gamma^{*}=\gamma^{R *}+\mathbf{i} \gamma^{I *}$ is the output solution corresponding to $w^{*}$. Furthermore, we suppose that the nonlinear function is continuous, that is, $\eta_{i}^{R}=\eta_{i}^{I}=0$ in formula (3.7). We obtain the following corollary, which is an extensional result of [57].

Corollary 3.5 The continuous complex-valued network (2.1) is globally exponentially stable to $w^{*}$ if

$$
\mu_{\mathcal{P}}(-D)+\|\bar{A}\|_{\mathcal{P}} \max \left\{\|\alpha\|_{\mathcal{P}},\|\beta\|_{\mathcal{P}}\right\}+\|\bar{B}\|_{\mathcal{P}} \max \left\{\|\alpha\|_{\mathcal{P}},\|\beta\|_{\mathcal{P}}\right\} \leq-\sigma<0 .
$$

\section{Stabilization result}

In this section, we design a set of state feedback controllers $m_{i}$ to make the solution of system (2.1) stable. Suppose $w^{*}$ is an equilibrium of (2.1) and $\gamma^{*}$ is the output solution corresponding to $w^{*}$. Then letting $\tilde{w}=w-w^{*}$ and $\tilde{\gamma}=\gamma-\gamma^{*}$, the control problem can be transformed into the following form:

$$
\dot{\tilde{w}}=-D \tilde{w}+A \tilde{\gamma}+B \tilde{\gamma}_{\tau}+m
$$

where $\tilde{\gamma}=\gamma-\gamma^{*}, \gamma \in K\left[h\left(w^{*}+\tilde{w}\right)\right]$, and $m=\left[m_{1}, m_{2}, \ldots, m_{n}\right]$ are feedback controllers.

Theorem 4.1 Assume that the discontinuous functions in (2.1) satisfy hypotheses A(1) and $A(2)$. Then the complex-valued network model is exponentially stable under the state feedback controllers $m_{i}=m_{i}^{R}+m_{i}^{I}$, where

$$
m_{i}^{R}=-\kappa \tilde{u}_{i}-\rho_{i} \operatorname{sgn} \tilde{u}_{i}, \quad m_{i}^{I}=-\kappa \tilde{v}_{i}-\rho_{i} \operatorname{sgn} \tilde{v}_{i}
$$

with

$$
\kappa>-\underline{d}+\bar{\xi}+\bar{\zeta} \text { and } \rho_{i}>\pi_{i}
$$

where $\xi_{i}=\max \left\{\sum_{j=1}^{n}\left\|a_{j i}\right\|_{1}\left\|\alpha_{j}\right\|_{1}, \sum_{j=1}^{n}\left\|a_{j i}\right\|_{1}\left\|\beta_{j}\right\|_{1}\right\}, \varsigma_{i}=\max \left\{\sum_{j=1}^{n}\left\|b_{j i}\right\|_{1}\left\|\alpha_{j}\right\|_{1}, \sum_{j=1}^{n}\|b\|_{1} \times\right.$ $\left.\left.\left\|\beta_{j}\right\|\right\}, \bar{\xi}=\max _{1 \leq i \leq n} \xi_{i}, \bar{\zeta}=\max _{1 \leq i \leq n} \varsigma_{i}, \pi_{i}=\max \left\{\sum_{j=1}^{n}\left\|a_{i j}\right\|_{1}\left\|\alpha_{j}\right\|_{1}, \sum_{j=1}^{n}\left\|b_{i j}\right\|_{1}\right)\left\|\eta_{j}\right\|_{1}\right\}$, and $\underline{d}=\min _{1 \leq i \leq n}\left\{d_{i}\right\}$.

Proof By separating all the parameters of system and the control inputs into their corresponding real and imaginary parts, system (4.1) can be expressed as follows:

$$
\left\{\begin{array}{l}
\dot{\tilde{u}}=-(D+\mathcal{K}) \tilde{u}+A^{R} \tilde{\gamma}^{R}-A^{I} \tilde{\gamma}^{I}+B^{R} \tilde{\gamma}_{\tau}-B^{I} \tilde{\gamma}_{\tau}^{I}-\rho \operatorname{sgn}(\tilde{u}), \\
\dot{\tilde{v}}=-(D+\mathcal{K}) \tilde{v}+A^{I} \tilde{\gamma}^{R}+A^{R} \tilde{\gamma}^{I}+B^{I} \gamma_{\tau}^{R}+B^{R} \tilde{\gamma}_{\tau}^{I}-\rho \operatorname{sgn}(\tilde{v}),
\end{array}\right.
$$

where $\mathcal{K}=\operatorname{diag}\{\kappa, \kappa, \ldots, \kappa\}, \rho=\operatorname{diag}\left\{\rho_{1}, \rho_{2}, \ldots, \rho_{n}\right\}, \operatorname{sgn}(\tilde{u})=\left[\operatorname{sgn}\left(\tilde{u}_{1}\right), \operatorname{sgn}\left(\tilde{u}_{2}\right), \ldots\right.$, $\left.\operatorname{sgn}\left(\tilde{u}_{n}\right)\right]^{\mathrm{T}}$, and $\operatorname{sgn}(\tilde{v})=\left[\operatorname{sgn}\left(\tilde{v}_{1}\right), \operatorname{sgn}\left(\tilde{v}_{2}\right), \ldots, \operatorname{sgn}\left(\tilde{v}_{n}\right)\right]^{\mathrm{T}}$. 
We choose a C-regular auxiliary function $W_{i}=\left|\tilde{u}_{i}\right|+\left|\tilde{v}_{i}\right|$. Based on the definitions of generalized gradient, for any $v_{i} \in \partial\left(\left|\tilde{u}_{i}\right|\right)$, we have $v_{i}=\operatorname{sgn}\left(\tilde{u}_{i}\right)$ if $\tilde{u}_{i} \neq 0$ and $v_{i}$ can be arbitrarily chosen in $[-1,1]$ if $\tilde{u}_{i}=0$. Especially, we choose $v_{i}=\operatorname{sgn}\left(\tilde{u}_{i}\right)$, and it can be seen that $v_{i} \tilde{u}_{i}=\left|\tilde{u}_{i}\right|$. By similar analysis we obtain $\vartheta_{i} \tilde{v}_{i}=\left|\tilde{v}_{i}\right|$. Calculating $\dot{W}_{i}$ along the trajectories of error system (4.4), we obtain

$$
\begin{aligned}
\dot{W}_{i}= & v_{i} \dot{\tilde{u}}_{i}+\vartheta_{i} \dot{\tilde{v}}_{i} \\
\leq & -\left(d_{i}+\kappa\right)\left|\tilde{u}_{i}\right|+\sum_{j=1}^{n}\left|a_{i j}^{R}\right|\left|\tilde{\gamma}_{j}^{R}\right|+\sum_{j=1}^{n}\left|a_{i j}^{I}\right|\left|\tilde{\gamma}_{j}^{I}\right|+\sum_{j=1}^{n}\left|b_{i j}^{R}\right|\left|\tilde{\gamma}_{j, \tau}^{R}\right| \\
& +\sum_{j=1}^{n}\left|b_{i j}^{I}\right|\left|\tilde{\gamma}_{j, \tau}^{I}\right|-\rho\left|\operatorname{sgn}\left(\tilde{u}_{i}\right)\right| \\
& -\left(d_{i}+\kappa\right)\left|\tilde{v}_{i}\right|+\sum_{j=1}^{n}\left|a_{i j}^{I}\right|\left|\tilde{\gamma}_{j}^{R}\right|+\sum_{j=1}^{n}\left|a_{i j}^{R}\right|\left|\tilde{\gamma}_{j}^{I}\right|+\sum_{j=1}^{n}\left|b_{i j}^{R}\right|\left|\tilde{\gamma}_{j, \tau}^{I}\right| \\
& +\sum_{j=1}^{n}\left|b_{i j}^{I}\right|\left|\tilde{\gamma}_{j, \tau}^{R}\right|-\rho\left|\operatorname{sgn}\left(\tilde{v}_{i}\right)\right| \\
\leq & -\left(d_{i}+\kappa\right)\left|\tilde{u}_{i}\right|+\sum_{j=1}^{n}\left|a_{i j}^{R}\right|\left(\alpha_{j}^{R}\left|\tilde{u}_{j}\right|+\beta_{j}^{R}\left|\tilde{v}_{j}(t)\right|+\eta_{j}^{R}\right)+\sum_{j=1}^{n}\left|a_{i j}^{I}\right|\left(\alpha_{j}^{I}\left|\tilde{u}_{j}\right|+\beta_{j}^{I}\left|\tilde{v}_{j}\right|+\eta_{j}^{I}\right) \\
& +\sum_{j=1}^{n}\left|b_{i j}^{R}\right|\left(\alpha_{j}^{R}\left|\tilde{u}_{j, \tau}\right|+\beta_{j}^{R}\left|\tilde{v}_{j, \tau}\right|+\eta_{j}^{R}\right)+\sum_{j=1}^{n}\left|b_{i j}^{I}\right|\left(\alpha_{j}^{I}\left|\tilde{u}_{j, \tau}\right|+\beta_{j}^{I}\left|\tilde{v}_{j, \tau}\right|+\eta_{j}^{I}\right)-\rho_{i}\left|\operatorname{sgn}\left(\tilde{u}_{i}\right)\right| \\
& -\left(d_{i}+\kappa\right)\left|\tilde{v}_{i}\right|+\sum_{j=1}^{n}\left|a_{i j}^{I}\right|\left(\alpha_{j}^{R}\left|\tilde{u}_{j}\right|+\beta_{j}^{R}\left|\tilde{v}_{j}\right|+\eta_{j}^{R}\right)\left|+\sum_{j=1}^{n}\right| a_{i j}^{R} \mid\left(\alpha_{j}^{I}\left|\tilde{u}_{j}\right|+\beta_{j}^{I}\left|\tilde{v}_{j}\right|+\eta_{j}^{I}\right) \\
& +\sum_{j=1}^{n}\left|b_{i j}^{R}\right|\left(\alpha_{j}^{I}\left|\tilde{u}_{j, \tau}\right|+\beta_{j}^{I}\left|\tilde{v}_{j, \tau}\right|+\eta_{j}^{I}\right)+\sum_{j=1}^{n}\left|b_{i j}^{I}\right|\left(\alpha_{j}^{R}\left|\tilde{u}_{j, \tau}\right|+\beta_{j}^{R}\left|\tilde{v}_{j, \tau}\right|+\eta_{j}^{R}\right)-\rho_{i}\left|\operatorname{sgn}\left(\tilde{v}_{i}\right)\right| \\
\leq & -(\underline{d}+\kappa)\left(\left|\tilde{u}_{i}\right|+\left|\tilde{v}_{i}\right|\right)+\xi_{i}\left(\left|\tilde{u}_{i}\right|+\left|\tilde{v}_{i}\right|\right)+s_{j}\left(\left|\tilde{u}_{i, \tau}\right|+\left|\tilde{v}_{i, \tau}\right|\right)-\left(\rho_{i}-\pi_{i}\right) \\
\leq & -(\underline{d}+\kappa) W_{i}+\xi_{i} W_{i}+s_{j} W_{i, \tau} .
\end{aligned}
$$

Letting $W=\sum_{i=1}^{n} W_{i}$, we obtain

$$
\begin{aligned}
\dot{W} & \leq-(\underline{d}+\kappa) \sum_{i=1}^{n} W_{i}+\bar{\xi} \sum_{i=1}^{n} W_{i}+\bar{\zeta} \sum_{i=1}^{n} W_{i, \tau} \\
& =-(\underline{d}+\kappa) W+\bar{\xi} W(t)+\bar{\zeta} W_{\tau} .
\end{aligned}
$$

Then by Lemma 2.9 and inequality (4.3) we obtain

$$
\left|\tilde{u}_{i}\right| \leq W \leq \sup _{-\infty \leq s \leq 0} W(s) e^{-\mu^{*} t}, \quad\left|\tilde{v}_{i}\right| \leq W \leq \sup _{-\infty \leq s \leq 0} W(s) e^{-\mu^{*} t},
$$

where $\mu^{*}$ is the solution of the equation $\mu-\underline{d}-\kappa+\bar{\xi}+\bar{\zeta} e^{\mu \tau}=0$. Therefore, complex-valued network (2.1) is exponentially stable under the designed controllers (4.2). 


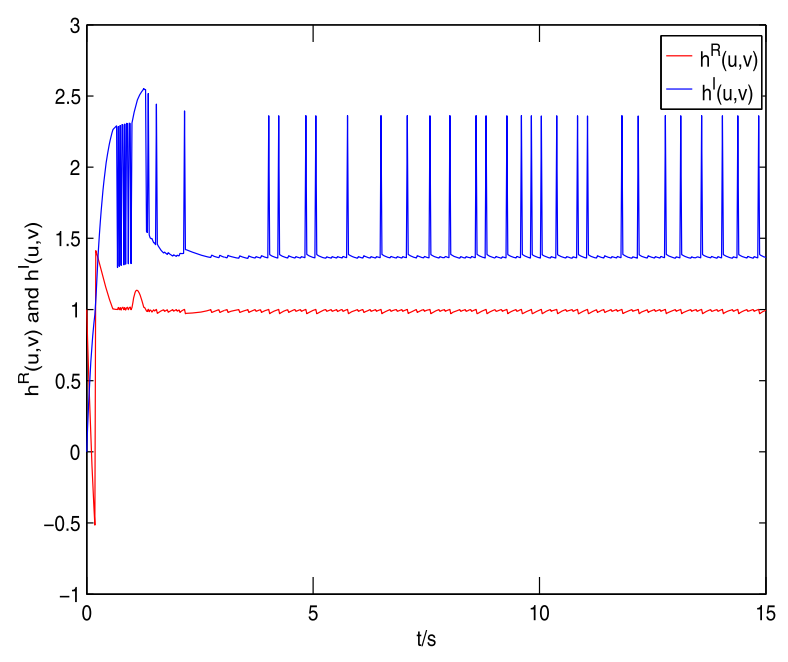

Figure 1 Time responses of the real part and imaginary part of $h(w)$ in Example 1

Remark 8 The inequality of $\kappa>-\underline{d}+\bar{\xi}+\bar{\zeta}$ in (4.3) is equivalent to formula (3.8) in Theorem 3.4 for $\mathcal{P}=1$. Therefore, the result of Theorem 4.1 is a direct application of Theorem 3.4 in the control filed.

\section{Numerical example}

We give two examples to demonstrate the correction of the obtained results.

Example 1 Consider the one-dimensional neural network model (2.1) with $D=2+2 \sqrt{3}$, $A=1+\mathbf{i} \sqrt{2}, B=\sqrt{2}+\mathbf{i}, J=1+\mathbf{i} \sqrt{5}$, and $\tau=1$. We choose the following activation function:

$$
h(w)= \begin{cases}w+1+\mathbf{i}, & \operatorname{Re}(w)>0, \operatorname{Im}(w)>0 \\ w-1, & \operatorname{Re}(w)<0, \operatorname{Im}(w)<0 \\ w+1, & \operatorname{Re}(w)<0, \operatorname{Im}(w)>0 \\ w-1+\mathbf{i}, & \operatorname{Re}(w)>0, \operatorname{Im}(w)<0\end{cases}
$$

and $h(0)=0$, which is shown in Fig. 1. From assumption $A(2)$ we obtain $\alpha=1, \beta=\sqrt{5}$, $\|A\|_{1}=\sqrt{3},\|B\|_{1}=\sqrt{3}$, and $\|J\|_{1}=\sqrt{6}$. According to Theorem 3.2, $\mu_{1}(-D)+\|A\|_{1}\|\alpha\|_{1}+$ $\|B\|_{1}\|\alpha\|_{1}=-2<0$ and $r=3 \sqrt{6}$, and it is easy to get that the invariant set is $\mathcal{S}=\left\{w \in \mathbb{C}^{n}\right.$ : $\left.\|w\|_{\mathcal{P}} \leq 3.67+\varepsilon\right\}$.

We separate $h(w)$ into its real and imaginary parts:

$$
h^{R}(u, v)=\left\{\begin{array}{ll}
u+1, & v>0, \\
u-1, & v<0,
\end{array} \quad \text { and } \quad h^{I}(u, v)= \begin{cases}v+1, & u>0, \\
v, & u<0 .\end{cases}\right.
$$

We obtain that $\alpha^{R}=1, \beta^{R}=0, \eta^{R}=2, \alpha^{I}=0, \beta^{I}=1$, and $\eta^{I}=1$ in (3.7). According to Theorem 3.4, it follows that $\mu_{1}(-D)=-(2+2 \sqrt{3}),\left\|A^{R}\right\|_{1}=1,\left\|A^{I}\right\|_{1}=\sqrt{2},\left\|B^{R}\right\|_{1}=\sqrt{2}$, $\left\|B^{I}\right\|_{1}=1,\left\|J^{R}\right\|_{1}=1$, and $\left\|J^{I}\right\|_{1}=\sqrt{5}$. Choosing $\sigma=2(\sqrt{3}-\sqrt{2})$ and $r=7+6 \sqrt{2}+\sqrt{5}$, it follows that the invariant set is $\mathcal{S}=\left\{w \in \mathbb{C}^{n}:\|\operatorname{Re}(w)\|_{1}+\|\operatorname{Im}(w)\|_{1} \leq 27.8+\varepsilon\right\}$. The 


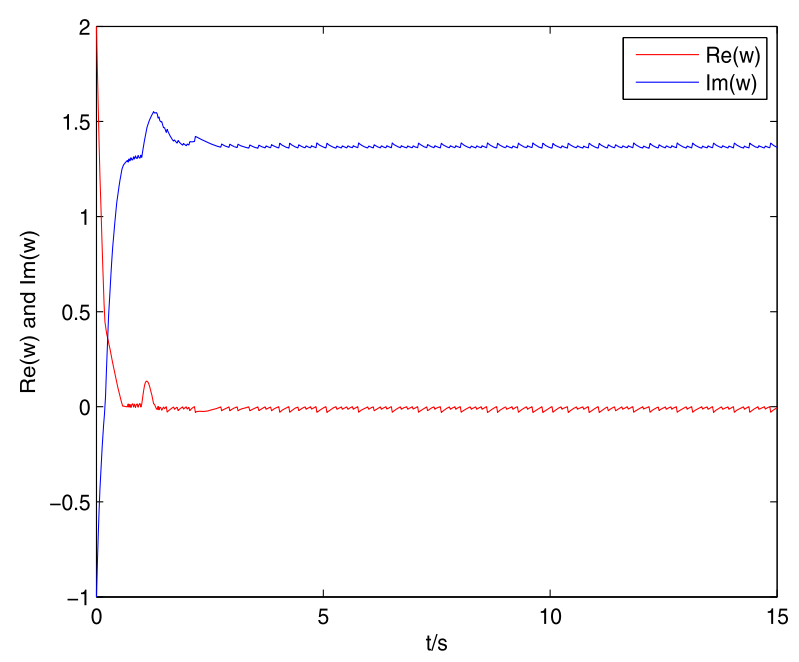

Figure 2 Time responses of the real part and imagine part of $w$ in Example 1

trajectories of real and imaginary parts of $v$ are shown in Fig. 2, and the invariant sets of Theorem 3.2 and Theorem 3.4 are shown in Fig. 3.

Remark 9 Obviously, from Fig. 3 we can see that the invariant set obtained from Theorem 3.2 is more accurate than that obtained in Theorem 3.4 because of the obvious inequality $\|w\|_{\mathcal{P}} \leq\|u\|_{\mathcal{P}}+\|v\|_{\mathcal{P}}$. Therefore the conditions of Theorem 3.2 are less conservative than those in Theorem 3.4 and greatly reduce the complexity of computation.

Example 2 Here we consider the neural network model with two complex neurons:

$$
\dot{w}=-D w+A h(w)+B h(w(t-\tau))
$$

where

$$
D=\left[\begin{array}{ll}
2 & 0 \\
0 & 1
\end{array}\right], \quad A=\left[\begin{array}{cc}
-1+0.5 \mathbf{i} & 1+0.5 \mathbf{i} \\
-0.5-\mathbf{i} & -1+0.5 \mathbf{i} \mathbf{i}
\end{array}\right], \quad B=\left[\begin{array}{cc}
2+\mathbf{i} & 1+0.5 \mathbf{i} \\
-0.5-2 \mathbf{i} & -1+0.5 \mathbf{i}
\end{array}\right],
$$

and $h_{i}(\chi)=[(\operatorname{Re}(\chi)-1) \operatorname{sgn}(\operatorname{Re}(\chi))+\operatorname{Im}(\chi)]+\mathbf{i}[(\operatorname{Im}(\chi)-1) \operatorname{sgn}(\operatorname{Im}(\chi))+\operatorname{Re}(\chi)]$ for any $\chi \in \mathbb{C}, i=1,2, \tau=1$. The trajectories for system (5.1) are shown in Fig. 4.

We separate $h(w)$ into its real part and parts:

$$
h_{i}^{R}\left(u_{i}, v_{i}\right)=\left(u_{i}-1\right) \operatorname{sgn}\left(u_{i}\right)+v_{i}, \quad h_{i}^{I}\left(u_{i}, v_{i}\right)=\left(v_{i}-1\right) \operatorname{sgn}\left(v_{i}\right)+u_{i} .
$$

We obtain that $\alpha_{i}^{R}=\beta_{i}^{R}=1, \eta_{i}^{R}=2, \alpha_{i}^{I}=\beta_{i}^{I}=1$, and $\eta_{i}^{I}=2$ for $i=1,2$. According to Theorem 4.1, it follows that $\underline{d}=1, \bar{\xi}=6, \bar{\zeta}=9$, and $\bar{\pi}=15$. If we choose $\kappa=15$ and $\rho_{i}=16$, then the equilibrium of (5.1) is exponentially stable under the designed controllers. The trajectories of the designed controllers and the state variables under control input are shown in Figs. 5 and 6, respectively. 


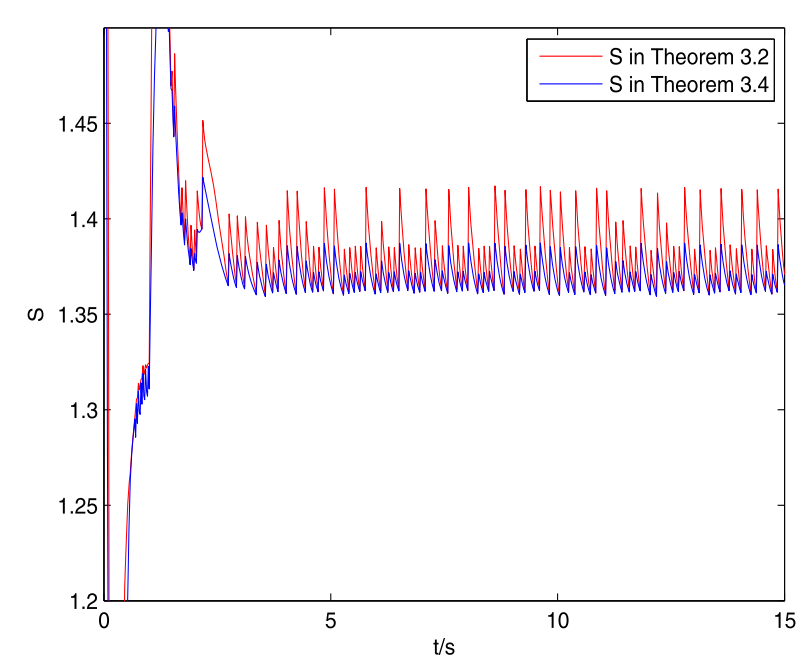

Figure 3 The positive invariant sets in Theorem 3.2 and Theorem 3.4 of Example 1

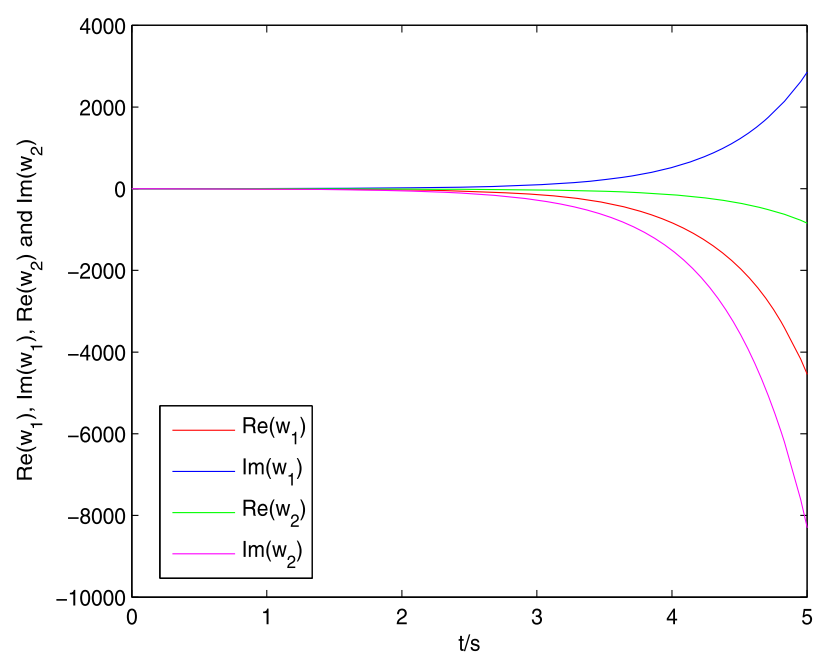

Figure 4 Time responses for the real and imaginary parts of the state solution in Example 2 without control input

\section{Conclusion}

In this paper, we investigate the global dissipativity and stabilization problem of discontinuous complex-valued networks via the matrix measure method. Compared with the existing results on continuous complex-valued neural networks, we propose a sufficient condition for the global dissipativity of discontinuous complex-valued neural networks based on differential inclusion. Compared with the results on discontinuous neural networks, we extend global dissipativity results to discontinuous neural networks with bivariate activation functions. In future, we will study the synchronization control of complex networks composed by discontinuous complex-valued differential equations. 


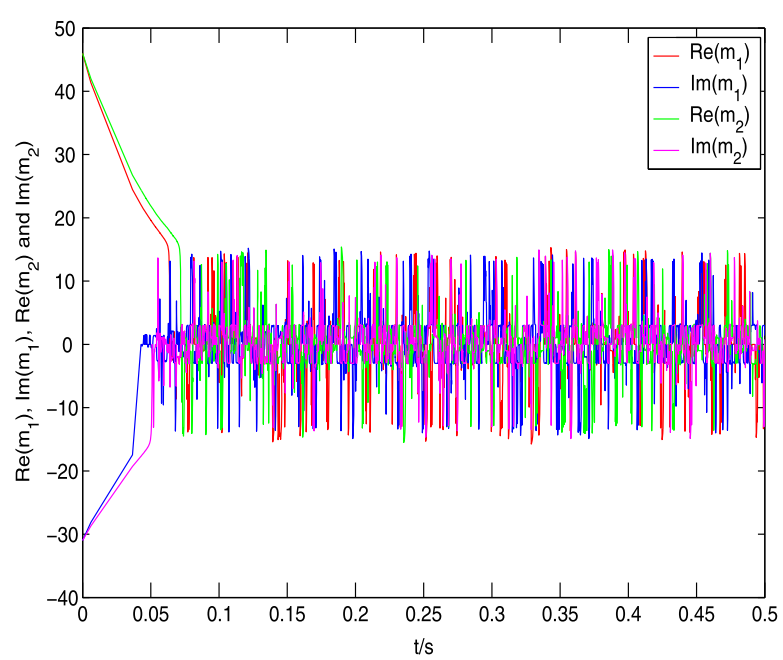

Figure 5 Time responses of the real and imaginary parts of the designed controllers in Example 2

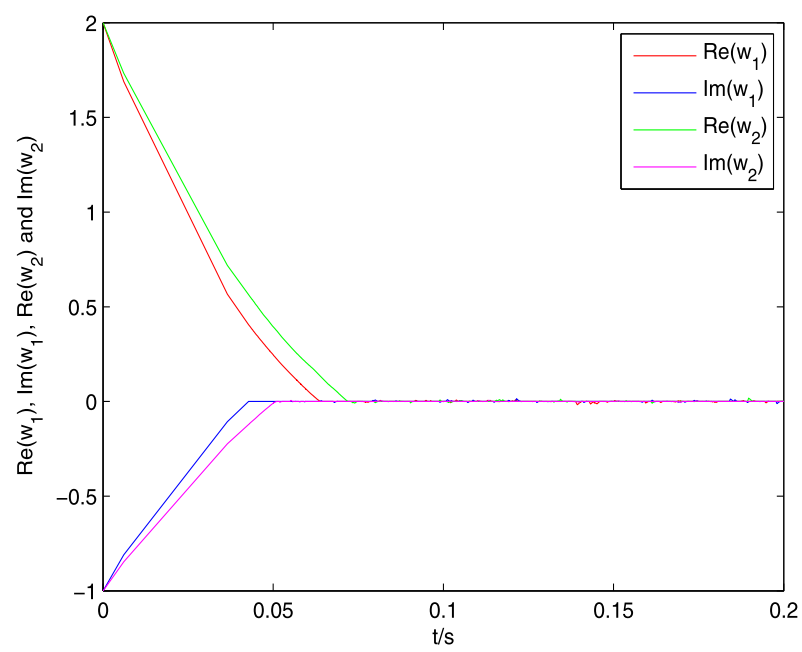

Figure 6 Time responses for the real and imaginary parts of the state solution in Example 2 under the designed controllers

Funding

This work was supported by National Natural Science Foundation of China Nos. 11601143, 61573003, 61573096, China Postdoctoral Science Foundation (No. 2018M632207), Key Project Supported by Scientific Research Fund of Hunan Provincial Education Department No. 15A038, and Jiangsu Provincial Key Laboratory of Networked Collective Intelligence under Grant No. BM2017002.

\section{Availability of data and materials}

We declare that materials described in the manuscript, including all relevant raw data, will be freely available to any scientist wishing to use them for noncommercial purposes, without breaching participant confidentiality.

\section{Competing interests}

We declare that there are no conflicts of interest with this work. We do not have any commercial or associative interest conflicts with the submitted work.

\section{Authors' contributions}

ZW carried out the stability analysis and controllers design. JC participated in the discussion and proposed the structure of this work. ZG was involved in drafting the manuscript and revising it critically. All authors read and approved the final manuscript. 


\section{Author details}

'School of Mathematics and Computational Science, Hunan First Normal University, Changsha, China. ${ }^{2}$ The Jiangsu Provincial Key Laboratory of Networked Collective Intelligence, and School of Mathematics, Southeast University, Nanjing, China. ${ }^{3}$ College of Mathematics and Econometrics, Hunan University, Changsha, China.

\section{Publisher's Note}

Springer Nature remains neutral with regard to jurisdictional claims in published maps and institutional affiliations.

Received: 2 May 2018 Accepted: 2 September 2018 Published online: 24 September 2018

\section{References}

1. Nitta, T.: Local minima in hierarchical structures of complex-valued neural networks. Neural Netw. 43, 1-7 (2013)

2. Tanaka, G., Aihara, K.: Complex-valued multistate associative memory with nonlinear multilevel functions for gray-level image reconstruction. IEEE Trans. Neural Netw. 20, 1463-1473 (2009)

3. Song, R.Z., Xiao, W.D., Zhang, H.G., Sun, C.Y.: Adaptive dynamic programming for a class of complex-valued nonlinear systems. IEEE Trans. Neural Netw. 25, 1733-1739 (2014)

4. Kawashima, K., Ogawa, T.: Complex-valued neural network for group-movement control of mobile robots. In: SICE Annual Conference, Akita, Japan (2012)

5. Zhang, S.C., Xia, Y.S., Zheng, W.X.: A complex-valued neural dynamical optimization approach and its stability analysis. Neural Netw. 61, 59-67 (2015)

6. Hirose, A.: Complex-Valued Neural Networks. Springer, Berlin (2012)

7. Nitta, T.: Complex-Valued Neural Networks: Utilizing High-Dimensional Parameters. Hershey, New York (2008)

8. Rudin, W: Real and Complex Analysis. Academic Press, New York (1987)

9. Hu, J., Wang, J.: Global stability of complex-valued recurrent neural networks with time delays. IEEE Trans. Neural Netw. Learn. Syst. 23, 853-865 (2012)

10. Zhang, Z.Y., Lin, C., Chen, B.: Global stability criterion for delayed complex-valued recurrent neural networks. IEEE Trans. Neural Netw. Learn. Syst. 25, 1704-1708 (2014)

11. Fang, T., Sun, J.T.: Further investigation on the stability of complex-valued recurrent neural networks with time delays. IEEE Trans. Neural Netw. Learn. Syst. 25, 1709-1713 (2014)

12. Zou, B., Song, Q.K.: Boundedness and complete stability of complex-valued neural networks with time delay. IEEE Trans. Neural Netw. Learn. Syst. 24, 1227-1238 (2013)

13. Rakkiyappan, R., Cao, J.D., Velmurugan, G.: Existence and uniform stability analysis of fractional-order complex-valued neural networks with time delays. IEEE Trans. Neural Netw. Learn. Syst. 26, 84-97 (2014)

14. Zhang, Z.Q., Yu, S.H.: Global asymptotic stability for a class of complex-valued Cohen-Grossberg neural networks with time delay. Neurocomputing 171, 1158-1166 (2015)

15. Velmurugan, G., Cao, J.D.: Further analysis of global stability of complex-valued neural networks with unbounded time-varying delays. Neural Netw. 67, 14-27 (2015)

16. Pan, J., Liu, X.Z.: Global exponential stability for complex-valued recurrent neural networks with asynchronous time delays. Neurocomputing 164, 293-299 (2015)

17. Liu, X.W., Chen, T.P.: Exponential stability of a class of complex-valued neural networks with time-varying delays. IEEE Trans. Neural Netw. Learn. Syst. 27, 593-606 (2016)

18. Wang, H.M., Duan, S.K., Huang, T.W., Wang, L.D., Li, C.D.: Exponential stability of complex-valued memristive recurrent neural networks. IEEE Trans. Neural Netw. Learn. Syst. 28, 766-771 (2016)

19. Li, X.D., Rakkiyappan, R., Velmurugan, G.: Dissipativity analysis of memristor based complex-valued neural networks with time-varying delays. Inf. Sci. 294, 645-665 (2014)

20. Song, Q.K., Yan, H., Zhao, Z.J., Liu, Y.R.: Global exponential stability of complex-valued neural networks with both time-varying delays and impulsive effects. Neural Netw. 79, 108-116 (2016)

21. Song, Q.K., Yan, H., Zhao, Z.J., Liu, Y.R.: Global exponential stability of impulsive complex-valued neural networks with both asynchronous time-varying and continuously distributed delays. Neural Netw. 81, 1-10 (2016)

22. Rakkiyappan, R., Sivaranjani, K., Velmurugan, G.: Passivity and passification of memristor based complex-valued recurrent neuralnet works with interval time varying delays. Neurocomputing 144, 391-407 (2014)

23. Kaslik, E., Radulescu, I.R.: Dynamics of complex-valued fractional-order neural networks. Neural Netw. 89, 39-49 (2017)

24. Hopfield, J.: Neurons with graded response have collective computational properties like those of two-state neurons Proc. Natl. Acad. Sci. USA 81, 3088-3092 (1984)

25. Tank, D., Hopfield, J.: Simple neural optimization networks: an A/D converter, signal decision circuit, and a linear programming circuit. IEEE Trans. Circuits Syst. I 33, 533-541 (1986)

26. Forti, M., Nistri, P.: Global convergence of neural networks with discontinuous neuron activations. IEEE Trans. Circuits Syst. I 50(11), 1421-1435 (2003)

27. Guo, Z.Y., Huang, L.H.: Generalized Lyapunov method for discontinuous systems. Nonlinear Anal. 71(7-8), 3083-3092 (2009)

28. Forti, M., Nistri, P.: Global exponential stability and global convergence in finite time of delayed neural network with infinite gain. IEEE Trans. Neural Netw. 16, 1449-1463 (2005)

29. Forti, M.: M-matrices and global convergence of discontinuous neural networks. Int. J. Circuit Theory Appl. 35 105-130 (2007)

30. Guo, Z.Y., Huang, L.H.: LMI conditions for global robust stability of delayed neural networks with discontinuous neuron activations. Appl. Math. Comput. 215, 889-900 (2009)

31. Guo, Z.Y., Huang, L.H.: Global output convergence of a class of recurrent delayed neural networks with discontinuous neuron activations. Neural Process. Lett. 30, 213-227 (2009)

32. Guo, Z.Y., Huang, L.H.: Global convergence of periodic solution of neural networks with discontinuous activation functions. Chaos Solitons Fractals 42, 2351-2356 (2009) 
33. Liu, X.Y., Chen, T.P., Cao, J.D., Lu, W.L.: Dissipativity and quasi-synchronization for neural networks with discontinuous activations and parameter mismatches. Neural Netw. 24, 1013-1021 (2011)

34. Yang, X.S., Cao, J.D.: Exponential synchronization of delayed neural networks with discontinuous activations. IEEE Trans. Circuits Syst. I 60(9), 2431-2439 (2013)

35. Huang, Y.J., Zhang, H.G., Wang, Z.S.: Multistability of complex-valued recurrent neural networks with real-imaginary-type activation functions. Appl. Math. Comput. 229, 187-200 (2014)

36. Liang, J.L., Gong, W.Q., Huang, T.W.: Multistability of complex-valued neural networks with discontinuous activation functions. Neural Netw. 84, 125-142 (2016)

37. Rakkiyappan, R., Cao, J.D., Velmurugan, G.: Multiple stability analysis of complex-valued neural networks with unbounded time-varying delays. Neurocomputing 149, 594-607 (2014)

38. Wang, Z.Y., Guo, Z.Y., Huang, L.H., Liu, X.Z.: Dynamical behavior of complex-valued Hopfield neural networks with discontinuous activation functions. Neural Process. Lett. 44, 1-23 (2017)

39. Brogliato, B., Lozano, R., Egeland, O., Maschke, B.: Dissipative Systems Analysis and Control: Theory and Applications. Springer, York (2000)

40. Wang, X., She, K., Zhong, S.M., Cheng, J.: On extended dissipativity analysis for neural networks with time-varying delay and general activation functions. Adv. Differ. Equ. 2016, 79 (2016)

41. Zeng, H.B., Teo, K.K., He, Y., Xu, H.L., Wang, W.: Sampled-data synchronization control for chaotic neural networks subject to actuator saturation. Neurocomputing 260, 25-31 (2017)

42. Zhang, C.K., He, Y., Jiang, L., Wu, M.: Stability analysis for delayed neural networks considering both conservativeness andComplexity. IEEE Trans. Neural Netw. Learn. Syst. 27(7), 1486-1501 (2016)

43. Arbia, A., Cao, J.D., Alsaedi, A.: Improved synchronization analysis of competitive neural networks with time-varying delays. Nonlinear Anal. 23(1), 82-102 (2018)

44. Arbi, A.: Dynamics of BAM neural networks with mixed delays and leakage time-varying delays in the weighted pseudo-almost periodic on time-space scales. Appl. Math. Comput. 219(17), 9408-9423 (2013)

45. Arbi, A., Aouiti, C., Chérif, F., et al.: Stability analysis for delayed high-order type of Hopfield neural networks with impulses. Neurocomputing 165, 312-329 (2015)

46. LV, X.X., Li, X.D.: Delay-dependent dissipativity of neural networks with mixed non-differentiable interval delays. Neurocomputing 267, 85-94 (2017)

47. Zhang, G.D., Zeng, Z.G., Hua, J.H.: New results on global exponential dissipativity analysis of memristive inertial neural networks with distributed time-varying delays. Neural Netw. 97, 183-191 (2018)

48. Zeng, H.B., He, Y., Shi, P., Wu, M., Xiao, S.P.: Dissipativity analysis of neural networks with time-varying delays. Neurocomputing $168,741-746$ (2015)

49. Zeng, H.B., Park, H., Zhang, C.F., Wang, W.: Stability and dissipativity analysis of static neural networks with interval time-varying delay. J. Franklin Inst. 352, 1284-1295 (2015)

50. Shi, P., Su, X.J., Li, F.B.: Dissipativity-based filtering for fuzzy switched systems with stochastic perturbation. IEEE Trans. Autom. Control 61(6), 1694-1699 (2016)

51. Zhang, Y.Q., Shi, P., Agarwal, R.K., Shi, Y.: Dissipativity analysis for discrete time-delay fuzzy neural networks with Markovian jumps. IEEE Trans. Fuzzy Syst. 24(2), 432-443 (2016)

52. Ahn, C.K., Shi, P.: Generalized dissipativity analysis of digital filters with finite-wordlength arithmetic. IEEE Trans. Circuits Syst. II, Express Briefs 63(4), 386-390 (2016)

53. Velmurugan, G., Rakkiyappan, R., Vembarasan, V.: Dissipativity and stability analysis of fractional-order complex-valued neural networks with time delay. Neural Netw. 86, 42-53 (2017)

54. Liu, X.Y., Fang, K.L., Liu, B.: A synthesis method based on stability analysis for complex-valued hop-field neural networks. In: Proc 7th Asian Control Conference, Hong Kong, pp. 1245-1250 (2009)

55. Ozdemir, N., Iskender, B.B., Ozgur, N.Y.: Complex-valued neural network with mobius activation function. Commun. Nonlinear Sci. Numer. Simul. 16, 4698-4703 (2011)

56. Cao, J.D., Wan, Y.: Matrix measure strategies for stability and synchronization of inertial BAM neural network with time delays. Neural Netw. 53, 165-172 (2014)

57. Gong, W.Q., Liang, J.L., Cao, J.D.: Matrix measure method for global exponential stability of complex-valued recurrent neural networks with time-varying delays. Neural Netw. 70, 81-89 (2015)

58. Fang, T., Sun, J.T.: Stability analysis of complex-valued nonlinear delay differential systems. Syst. Control Lett. 62 910-914 (2013)

59. Aubin, J.P., Cellina, A.: Differential Inclusions. Springer, Berlin (1984)

60. Cai, Z.W., Huang, L.H.: Functional differential inclusions and dynamic behaviors for memristor-based BAM neural networks with time-varying delays. Commun. Nonlinear Sci. Numer. Simul. 19, 1279-1300 (2014)

61. Filippov, A.F.: Differential Equations with Discontinuous Right-Hand Side. Kluwer Academic, Boston (1988)

62. Wang, J.F., Huang, L.H., Guo, Z.Y.: Dynamical behavior of delayed Hopfield neural networks with discontinuous activations. Appl. Math. Model. 33, 1793-1802 (2009) 